\title{
INVESTIGACIONES
}

\section{Pensamiento sobre la formación ciudadana en estudiantes universitarios, dimensiones para la construcción de un cuestionario}

\author{
Thinking about citizen education in university students, \\ dimensions for the construction of a questionnaire \\ Pensando na formação cidadã de universitários, \\ dimensões para a construção de um questionário
}

\author{
Natalia Contreras Quiroz ${ }^{a}$ \\ ${ }^{a}$ Universidad Católica Silva Henríquez, Facultad de Educación, \\ Escuela de Educación en Historia y Geografía. Chile. \\ ncontreras@ucsh.cl
}

\begin{abstract}
RESUMEN
Teniendo en cuenta que desde 2016 existe una ley de formación ciudadana en Chile, el objetivo de este trabajo es dar a conocer resultados parciales de una investigación de carácter cuantitativo financiada por la Universidad Católica Silva Henríquez ${ }^{1}$, principalmente las dimensiones conceptuales del modelo teórico utilizado para la construcción de un cuestionario y el resultado de la evaluación de contenido realizada a través de la validación de jueces expertos. La ciudadanía se construye culturalmente, por lo que es importante identificar lo que los jóvenes entienden y cómo proyectan su papel como educadores, así como su contribución a la sociedad en este ámbito. La formación de la ciudadanía es un área de conocimiento que forma parte del sistema educativo tanto como las disciplinas de las ciencias sociales y naturales que, se integran en el plan de estudios según lo indican las tendencias paradigmáticas de cada sociedad y su contexto. La metodología de trabajo es cuantitativa, mediante un cuestionario, con validación de contenidos y validación de constructos. El modelo teórico propuesto se construye mediante categorías de análisis asociadas al conocimiento del sistema político del país, el desarrollo de competencias y habilidades prosociales y la educación en valores. Esta ponencia desarrolla el proceso de validación de contenido del cuestionario a través de jueces expertos, puesto que la investigación continúa en proceso.
\end{abstract}

Palabras claves: Educación ciudadana, Educación política, Ciudadanía, Formación inicial de profesores.

\begin{abstract}
Taking into account that since 2016 there is a law on citizen training in Chile, the objective of this work is to present partial results of a quantitative research financed by the Universidad Católica Silva Henríquez, mainly the conceptual dimensions of the theoretical model used for the construction of a questionnaire and the result of the content evaluation carried out through the validation of expert judges. Citizenship is culturally constructed, so it is important to identify what young people understand and how they project their role as educators, as well as their contribution to society in this area. Citizenship formation is an area of knowledge that is as much a part of the educational system as the disciplines of the social and natural sciences that are integrated into the curriculum as indicated by the paradigmatic trends of each society and its context. The work methodology is quantitative, through a questionnaire, with content validation and construct validation. The proposed theoretical model is constructed through categories of analysis associated with knowledge of the country's political system,
\end{abstract}

Adjudicación Proyecto Modalidad Semilla 2020 código S - 2002NCQ, Universidad Católica Silva Henríquez, Chile. 
the development of prosocial competencies and skills, and education in values. This paper develops the process of content validation of the questionnaire through expert judges, since the research is still in progress.

Key words: Citizenship education, Political education, Citizenship, Initial teacher training.

\section{RESUMO}

Levando em consideração que desde 2016 existe uma lei de formação cidadã no Chile, o objetivo deste trabalho é apresentar resultados parciais de uma pesquisa quantitativa financiada pela Universidade Católica Silva Henríquez, principalmente as dimensões conceituais do modelo teórico utilizado para o construção de questionário e resultado da avaliação de conteúdo realizada por meio da validação por juízes especialistas. A cidadania é construída culturalmente, por isso é importante identificar o que os jovens entendem e como projetam o seu papel de educadores, bem como a sua contribuição para a sociedade nesta área. A formação da cidadania é uma área do conhecimento que faz parte do sistema educacional, assim como as disciplinas das ciências sociais e naturais que se integram ao currículo indicadas pelas tendências paradigmáticas de cada sociedade e seu contexto. A metodologia de trabalho é quantitativa, por meio de questionário, com validação de conteúdo e validação de construto. O modelo teórico proposto é construído por meio de categorias de análise associadas ao conhecimento do sistema político do país, ao desenvolvimento de competências e habilidades pró-sociais e à educação em valores. Esta apresentação desenvolve o processo de validação do conteúdo do questionário por meio de juízes especialistas, uma vez que a investigação ainda está em andamento.

Palavras-chave: Educação para a cidadania, Educação política, Cidadania, Formação inicial de profesores.

\section{INTRODUCCIÓN}

La educación de la ciudadanía se presenta como una dimensión de la enseñanza compleja para cualquier sistema educativo, puesto que refiere unos modos concretos de creación de cultura política, y por consecuencia se transforma en un pilar clave para la protección de la vida democrática. La experiencia, nacional e internacional permite identificar al menos dos tendencias en la formación de la ciudadanía que surge de las políticas públicas y que se insertan en el currículum, por una parte, el enfoque minimalista que se concentra en un tipo de participación mínima, restringido básicamente al sufragio y al conocimiento de leyes y otros deberes normativos. Por otra parte, el enfoque maximalista fomenta una participación activa de los ciudadanos, que va más allá de hitos como el voto, puesto que también apela al compromiso social y el ejercicio de acciones de cambio (Cox et al., 2014).

El estudio Educación ciudadana en América Latina: prioridades de los currículos escolares (Cox et al., 2014) mediante un análisis comparado de 6 países de la región (Colombia, Chile, Guatemala, México, Paraguay, y República Dominicana) indagó en el equilibrio en términos de presencia temática en los currículos de: derechos y obligaciones civiles, participación ciudadana y sistema político. Uno de los resultados más significativos señala que existe un énfasis en los aspectos de la vida civil, más que en el desarrollo de un sistema cívico participativo:

Los currículos, de acuerdo a nuestro análisis de los contenidos de mayor o menor presencia en los mismos, no privilegian el dominio (político) en que confluyen todos los grupos y comunidades para procesar sus intereses, y en que institucionalmente se elaboran las definiciones del bien común y se procesan las reglas del orden societal en su conjunto. 
A diferencia de este desafío en la región latinoamericana, la comunidad europea enfrenta una fuerte diversificación de modos de enseñanza de la formación ciudadana, un estudio supranacional y comparado realizado en la Unión Europea (Cortés et al., 2016), permitió identificar la necesidad de crear elementos comunes a todos los países de la Unión, "para así construir una base que fomente una ciudadanía europea basada en la fraternidad y la solidaridad sin que esto sea en detrimento de la identidad nacional de cada uno de los países miembros" (Cortés et al., 2016, p. 190).

Con el retorno a la democracia en la década del 90, Chile da un giro desde el enfoque minimalista a uno maximalista, proceso que se consolida en 2016 cuando se promulga la Ley 20.911, que establece la obligatoriedad de que todos los establecimientos educacionales del país cuenten con un Plan de Formación Ciudadana. Además, en el segundo artículo se señaló un proceso de reforma curricular que integraba el curso de Educación Ciudadana en los niveles de tercer y cuarto año de enseñanza media. Los planes de formación ciudadana comenzaron su implementación el mismo 2016 y la reforma curricular se integró al sistema escolar en 2019.

El Plan de Formación Ciudadana corresponde a un plan normativo que debe tener cada establecimiento, en el que se describen acciones de educación ciudadana que pueden estar asociadas a disciplinas específicas de formación, pueden ser transversales a la comunidad educativa, estar centrada en actores concretos e incluso ser de vinculación con el medio. Estas acciones responden a nueve objetivos de formación ciudadana que están contenidos en la Ley 20.911 y abordan dimensiones tanto conceptuales, como actitudinales y valórica, a la vez que promueven competencias como el pensamiento crítico y la participación democrática. Se espera que cada comunidad educativa de manera participativa y democrática establezca cuales son los objetivos que más se alinean con el proyecto institucional, los sellos identitarios y las propias necesidades y propósitos que se tengan para la formación ciudadana. Este plan normativo es responsabilidad tanto de encargados de la gestión escolar como de la comunidad de profesores, estudiantes y apoderados, según sea establecido por el mismo plan y validado por el Consejo Escolar de cada establecimiento.

En 2018 el Programa de las Naciones Unidas para el Desarrollo a través del "Estudio sobre la puesta en marcha del Plan de Formación Ciudadana" en Chile informó que:

El 34\% de los establecimientos incluye en sus planes alguna acción relacionada con un acto, una feria o celebración. Dentro de esta categoría se incluyen con frecuencia actividades que celebran el día de distintos estamentos o temáticas (el día del alumno, del profesor, del asistente de la educación, de la madre, de la convivencia, entre otros) o la celebración de determinadas efemérides a través de actos cívicos. En este código también se describen celebraciones culturales y artísticas (festivales de la canción, desfiles culturales, exposiciones artísticas) y actividades de corte más institucional de los establecimientos (como aniversarios y graduaciones). Por último, se mencionan actividades que celebran las fiestas patrias o el folclore chileno, así como actividades que celebran la interculturalidad (PNUD, 2018, p. 31).

Estas actividades que concentran la tipología de acciones más recurrentes sobre formación ciudadana se caracterizan por tener un carácter episódico y extracurricular, su principal foco está puesto sobre la convivencia, la identidad y pertenencia a la comunidad educativa, lo que sin duda es fundamental para la educación ciudadana, pero es solo 
una de sus múltiples dimensiones. El gráfico 1, que contiene información del mismo estudio, evidencia los espacios de la escuela en que se desarrollan con mayor o menor concentración las acciones de formación ciudadana, como se puede observar, las actividades extraprogramáticas son las más dominantes y quedan desatendidas aquellas asociadas a la vinculación con el medio y el espacio curricular.

Figura 1. Espacios en los que se desarrollan actividades de Formación Ciudadana

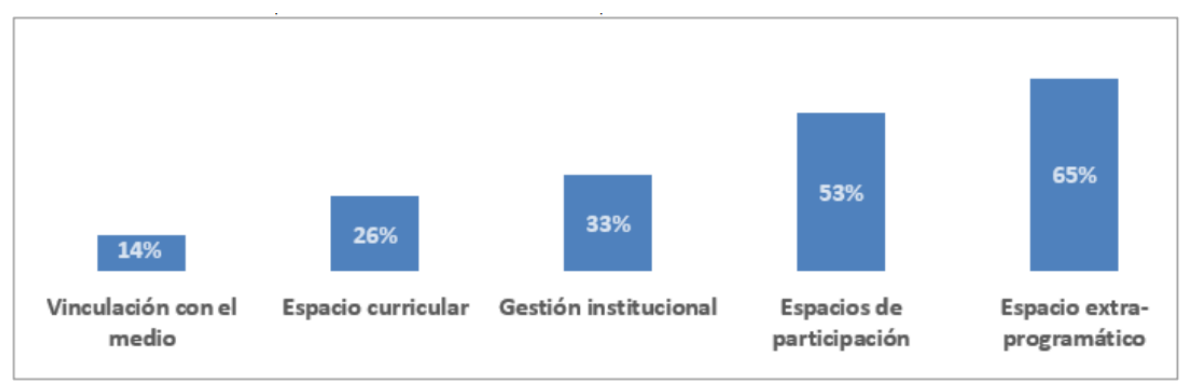

Fuente: (PNUD, 2018).

La relevancia de estos resultados se asocia a la transversalidad en los niveles de enseñanza que debe tener el Plan de Formación Ciudadana, puesto que según establece la Ley 20.911 debe abarcar a la Educación de Párvulos, la Educación Básica y la Educación Media. Y si bien existe una asignatura específica de Educación Ciudadana para tercero y cuarto año de enseñanza media, esto es insuficiente para una formación integral y puede quedar invisibilizado al resolverse solo mediante acciones episódicas, asociadas a efemérides y celebraciones con temas muy concretos. Un enfoque similar arroja un estudio realizado por Orella y Muñoz (2019) que indagó en las concepciones y representaciones sobre la ciudadanía que poseen diversos actores de colegios municipales ubicados en dos regiones del sur del Chile, uno de los principales resultados señala que a pesar de la política pública sobre formación ciudadana que existe en el país persiste en la dimensión de un ciudadano ligado a derechos y deberes y un sentido marcadamente nacional.

Entre los variados desafíos que tiene la implementación de esta ley en el país, el estudio del PNUD señala la preparación en formación ciudadana, a nivel de contenidos y didáctica como un asunto que requiere de apoyo con apremio (PNUD, 2018), puesto que la preparación técnica y profesional es clave para que la educación de la ciudadanía logre desplegar sus objetivos con efectividad y solo como un plan normativo nominal.

Como se ha mencionado, los objetivos de formación ciudadana de la ley se ven reforzados por la integración al currículum nacional de una asignatura específica de Educación Ciudadana en los últimos años de formación escolar, específicamente tercer y cuarto año medio, los contenidos que se abordan pueden identificarse inicialmente a través de las unidades temáticas de cada curso. 
Tabla 1. Unidades temáticas del curso Educación ciudadana

\begin{tabular}{|l|l|}
\hline \multicolumn{1}{|c|}{$\mathbf{3}^{\circ}$ medio } & \multicolumn{1}{c|}{$\mathbf{4}^{\circ}$ medio } \\
\hline Unidad 1: Estado, democracia y ciudadanía & $\begin{array}{l}\text { Unidad 1: La participación ciudadana } \\
\text { contribuye con soluciones a los desafíos, } \\
\text { problemas y conflictos presentes en la sociedad }\end{array}$ \\
\hline Unidad 2: Justicia y derechos humanos & $\begin{array}{l}\text { Unidad 2: Medios de comunicación masivos, } \\
\text { ciudadanía responsable y ética para una } \\
\text { sociedad democrática }\end{array}$ \\
\hline $\begin{array}{l}\text { Unidad 3: Participación y la organización } \\
\text { territorial en democracia }\end{array}$ & $\begin{array}{l}\text { Unidad 3: Principios éticos para orientar la } \\
\text { vida en democracia }\end{array}$ \\
\hline Unidad 4: Relaciones entre Estado y mercado & $\begin{array}{l}\text { Unidad 4: Modelos de desarrollo económico y } \\
\text { derechos laborales, decisiones para construir la } \\
\text { sociedad que queremos }\end{array}$ \\
\hline
\end{tabular}

Fuente: (Curriculum nacional, s. f.).

Como se puede observar, las temáticas abordadas aluden a dimensiones de enseñanza que son complejas, que involucran un conocimiento interdisciplinar de la sociedad, vistos en gran parte a través de las ciencias humanas o sociales, lo que favorece una educación integral y se complementa coherentemente con los objetivos de formación ciudadana de la Ley 20.911, pero que dista mucho con las principales acciones que se desarrollan en los establecimientos educacionales.

La formación ciudadana, de esta manera, es un ámbito del conocimiento que es parte del sistema educativo y, por lo tanto, un contenido conceptual, procedimental y actitudinal, que puede ser sujeto de procesos didácticos y evaluativos. Este contexto interpela directamente a los programas de formación del profesorado debido a que deben dar respuesta a las demandas y necesidades de preparación profesional de sus estudiantes en temas concretos de educación de la ciudadanía, es decir, sus procesos pedagógicos (Contreras y Sánchez, 2020, p. 338).

En el caso de Chile, esta interpelación impacta la formación pedagógica de todas las disciplinas, pero con especial énfasis a la educación de párvulos, enseñanza general básica y educación en historia, geografía y ciencias sociales, ya que son las áreas que tienen asignada más responsabilidad a la hora de llevar acciones de formación ciudadana o de lleno, como en el caso de historia, geografía y ciencias sociales, son estas y estos los profesionales a los que les corresponde la enseñanza de la educación ciudadana durante los dos años anteriores a que los estudiantes cumplan la mayoría de edad y puedan conseguir su ciudadanía jurídica.

En este contexto surgió la inquietud por identificar y comprender el pensamiento que estudiantes de pedagogía tienen sobre la educación de la ciudadanía, principalmente porque los profesores que están en el sistema ya tienen una formación profesional que se adapta a los requerimientos del medio, como es el surgimiento de la Ley 20.911, pero aquellos estudiantes que entran a su formación inicial docente están en plena fase de conocimiento 
e integración a un ámbito educativo innovado. La educación de la ciudadanía ha estado siempre presente en el sistema educativo chileno, pero desde el año 2016 se presenta con nuevos objetivos y contenidos.

Esta investigación se realiza con el patrocinio de fondos internos de la Universidad Católica Silva Henríquez y busca obtener sus resultados a través de un cuestionario de carácter cuantitativo, aplicado a estudiantes de primer año de las carreras de: Educación de párvulos, Educación general básica y Educación en historia y geografía. Actualmente los resultados del cuestionario están en proceso de sistematización y serán presentados en una próxima publicación.

El objetivo de este trabajo es dar a conocer resultados parciales de dicho estudio, principalmente dar a conocer las dimensiones conceptuales utilizadas para la construcción del cuestionario y el resultado de la evaluación de contenido realizada a través de la validación de jueces expertos.

\section{METODOLOGÍA}

Esta investigación cuantitativa no experimental de carácter exploratorio consideró un proceso de revisión de literatura teórica como también de experiencias sobre estudios de formación ciudadana e incluso sistemas de medición. Posteriormente, se trabajó en el diseño del cuestionario que fue validado con jueces expertos para ser aplicado a los estudiantes del proyecto de investigación.

\subsection{FASE 1. REVISIÓN DE LITERATURA, INSTRUMENTOS DE MEDICIÓN Y POLÍTICA PÚBLICA VIGENTE SOBRE FORMACIÓN CIUDADANA EN CHILE}

Para comprender la actual política pública sobre formación ciudadana en Chile, se realizó una pesquisa en contenido histórico (Cox y Castillo, 2015; Fierro, 2016; Mardones, 2018; Serrano, 2018) explicativo que permite evidenciar que la educación de la ciudadanía ha sido un tema de interés desde los orígenes del Estado, puesto que se le adjudica gran responsabilidad en la composición de una identidad nacional y de valoración de lo patrio. A su vez, se puede identificar el cambio de foco que tiene este enfoque educativo en el periodo posterior a la dictadura cívico - militar en Chile, donde el interés por los procesos democráticos, la defensa de los Derechos Humanos, la valoración de la diversidad y la protección del medioambiente se tornan tan importantes como el carácter institucional y nacionalista que había caracterizado anteriormente a la educación cívica. Actualmente, estas temáticas de educación de la ciudadanía conviven en el sistema educativo chileno, porque están contenidos en la Ley 20.911, pero sobre todo dependiendo en gran medida de los énfasis formativos de quienes la enseñan.

También fue de interés identificar instrumentos de medición para el conocimiento sobre educación ciudadana, puesto que son una referencia para poder diseñar un instrumento aplicable a estudiantes en procesos formativos de una carrera profesional en pedagogía. Específicamente en Chile, la formación ciudadana está siendo evaluada a través del Sistema de Medición de la Calidad de la Educación, perteneciente a la Agencia de Calidad, esto se realiza mediante el Cuestionario de indicadores de desarrollo social y personal que incluye la dimensión de "Participación y Formación Ciudadana" (Agencia de la Calidad 
de la Educación, 2019), el indicador considera las actitudes de los estudiantes frente a su establecimiento y sus percepciones en relación al nivel en que la institución fomenta la participación democrática en la comunidad escolar.

Otros referentes utilizados para la construcción del cuestionario son los que emanan de estudios internacionales, específicamente de la Agencia Internacional para la Evaluación del Rendimiento Educativo, institución que evalúa mediante instrumentos métricos contextos, implementación e impacto de políticas públicas. En el caso de la formación ciudadana, hay varios estudios que incluyen a Chile como objeto de investigación y que describen el contexto anterior a la implementación de la Ley 20.911 (Agencia de Calidad de la Educación, 2015; Schulz, Ainley et al., 2010b, 2010a; Schulz, Fraillon et al., 2010).

Junto con los referentes de medición se trabajó en la construcción de un modelo teórico sobre formación ciudadana que integrara dimensiones provenientes de la comunidad académica tanto del ámbito de la educación como de la filosofía, sobre todo para identificar las relaciones entre la acción política y la educación (Chaux et al., 2012; Cortina, 2009; Cox y Castillo, 2015; Dewey, 2003; Estévez y Menéndez, 2017; Fierro, 2016; Pérez, 2016; Rancière, 2014; Savater, 2007; Touraine, 2015). Además, de revisó la literatura empírica sobre formación ciudadana en el contexto chileno (Castillo Riquelme et al., 2018; Contreras y Sánchez, 2020; Cox y Castillo, 2015; Magendzo y Pavez, 2016; Mardones, 2018; Núñez, 2017) como también estudios sobre educación de la ciudadanía en el espacio universitario (Leung y Chan, 2019; Nurdin, 2017; Parji y Feriandi, 2020).

\subsection{FASE 2. CONSTRUCCIÓN DEL MODELO TEÓRICO Y VALIDACIÓN DE CONTENIDO A TRAVÉS DE JUECES EXPERTOS}

Considerando la revisión de literatura que permitió identificar referentes de instrumentos de medición y también la construcción de un modelo teórico se diseñó el Cuestionario de Pensamiento en Estudiantes Universitarios sobre Formación Ciudadana (PEU-FC). El contenido del cuestionario se elaboró a partir de una matriz de operacionalización que distinguió el objetivo del instrumento, la definición del constructo, dimensiones, indicadores y/o ítems (Corral, 2010; Ruiz, 2014).

Cabe destacar que una de las investigaciones relevantes para la construcción del modelo teórico surgió de un estudio previo, de carácter cualitativo realizado mediante análisis de contenido. Dicha investigación titulada "Formación ciudadana: significados emergentes en contexto de crisis social" (Contreras y Sánchez, 2020) también se realizó en contexto de educación superior y se trabajó desde la elaboración de categorías de análisis apriorísticas (competencias y habilidades cívicas, conocimiento cívico y valores en formación ciudadana) y emergentes (emociones y función pedagógica de la formación ciudadana).

El objetivo del instrumento es identificar y caracterizar el pensamiento que estudiantes universitarios tienen sobre la formación de la ciudadanía, a partir de las dimensiones: Conocimiento Cívico, Competencias y habilidades cívicas, Valores en Formación Ciudadana y Educación del profesorado en Formación Ciudadana.

Las preguntas del cuestionario se elaboraron mediante ítems con respuesta tipo escala. Una vez construidos los ítems y diseñado el instrumento psicométrico, se realizó la validez de contenido a través de jueces expertos, se consultó a 10 expertos seleccionados según su perfil profesional y académico. 
Los expertos evaluaron cada ítem de acuerdo a su pertinencia con el marco teórico/ conceptual, redacción, pertinencia a la población objetivo, etc. Estos puntuaran cada pregunta valorándola como esencial con 3 punto, adecuada con reparos con 2 puntos, y rechazo con 1 puntos. El Índice de Validez de Contenido (IVC) se analizó por medio del coeficiente de Lawshe (Lawshe, 1975), debido a que éste tiene la ventaja de entregar un coeficiente único de validez para cada ítem, con un nivel mínimo de aprobación asociado a la probabilidad de un acuerdo debido al azar entre los jueces.

\subsection{APROXIMACIONES TEÓRICAS A UN MODELO DE FORMACIÓN CIUDADANA}

Un modelo de formación ciudadana que distinga el contexto global y las necesidades locales de las sociedades comienza por el reconocimiento de su rol en la búsqueda de la justicia social, por lo tanto, se posiciona en una idea de ciudadanía activa, enmarcada por la educación para una ciudadanía democrática y con enfoque de Derechos Humanos.

Ser ciudadana o ciudadano, no es una condición innata del ser humano, es de hecho una categoría que se construye y que también implica un sentido de pertenencia en los miembros de una comunidad:

No hay democracia sin conciencia de pertenencia a una colectividad política, una nación en la mayoría de los casos, pero también una comuna, una región y hasta un conjunto federal. La democracia se asienta sobre la responsabilidad de los ciudadanos de un país. Si éstos no se sienten responsables de su gobierno, porque éste ejerce su poder en un conjunto territorial que les parece artificial o ajeno, no puede haber representatividad de los dirigentes ni libre elección de éstos por los dirigidos (Touraine, 2015, p. 99).

Por este motivo, es que se espera de la formación ciudadana que sea una disciplina que desarrolle competencias y habilidades ciudadanas, que reflexione críticamente sobre el conocimiento y eduque en valores que cuestionen el status quo de las sociedades, para dar cuenta de la desigualdad, y así la escuela pueda ser un espacio de emancipación y no de reproducción de las injusticias. Tal como lo plantean Bourdieu y Passeron (1996):

En una sociedad en la que la obtención de los privilegios sociales depende cada vez más estrechamente de la posesión de títulos escolares, la escuela no tiene por función únicamente asegurar la sucesión discreta de los derechos de la burguesía que ya no pueden seguir transmitiéndose de una manera directa y declarada (p. 269).

Pérez (2016) también pone énfasis en los aspectos jurídicos y de identidad de pertenencia de la ciudadanía, puesto que la vincula directamente al desarrollo de la democracia, entendida como un sistema de gobierno en el que los individuos tienen una serie de derechos que deben ser respetados por los gobernantes, los que a vez están obligados a cumplir las leyes y normas de la comunidad de pertenencia. Con esta lógica la formación de ciudadanos se centra en la preparación de las jóvenes generaciones para vivir y ejercer el oficio de ciudadano o ciudadana en una comunidad configuradora de la nación. "La misión fundamental de la escuela es la integración y socialización política de los individuos en una comunidad de ciudadanos capaces de convivir en el espacio público" (Pérez, 2016, p. 122). 
Evidentemente, la naturaleza de la ciudadanía moderna exige una relación política del individuo con su comunidad, esa interacción constituye un doble proceso de aprendizaje, que Peña (en Cox y Castillo, 2015) identifica como ciudadanía por titularidad y ciudadanía por membresía. La primera dimensión corresponde a los derechos que habilitan al individuo para la participación política, lo que en el contexto chileno es dado por la Constitución Política de Estado. La ciudadanía por membresía es una segunda dimensión en la que se da por hecho el aprendizaje que el individuo ha hecho de las normas legales de su carácter de ciudadano, que es capaz de identificar sus posibilidades de participación política. La membresía indica un nivel de involucramiento mayor a partir de esa adquisición de derecho, como la pertenencia a la comunidad donde esas decisiones se adoptan. Es lo que Adela Cortina llama ciudadanía por identidad: "un concepto pleno de ciudadanía integra un status legal (un conjunto de derechos), un status moral (un conjunto de responsabilidades) y también una identidad, por la que una persona se sabe y siente perteneciente a una sociedad" (Cortina, 2009, p. 151).

La ciudadanía jurídica, dada por los marcos legales de cada comunidad, transciende a esta dimensión, puesto que también se despliega en otros ámbitos de la vida en comunidad, del espacio público o del bien común. Cortina (2009) distingue varios tipos más de ciudadanía que en conjunto describen a los ciudadanos del mundo.

Figura 1. Modelo de ciudadanía propuesto por Adela Cortina

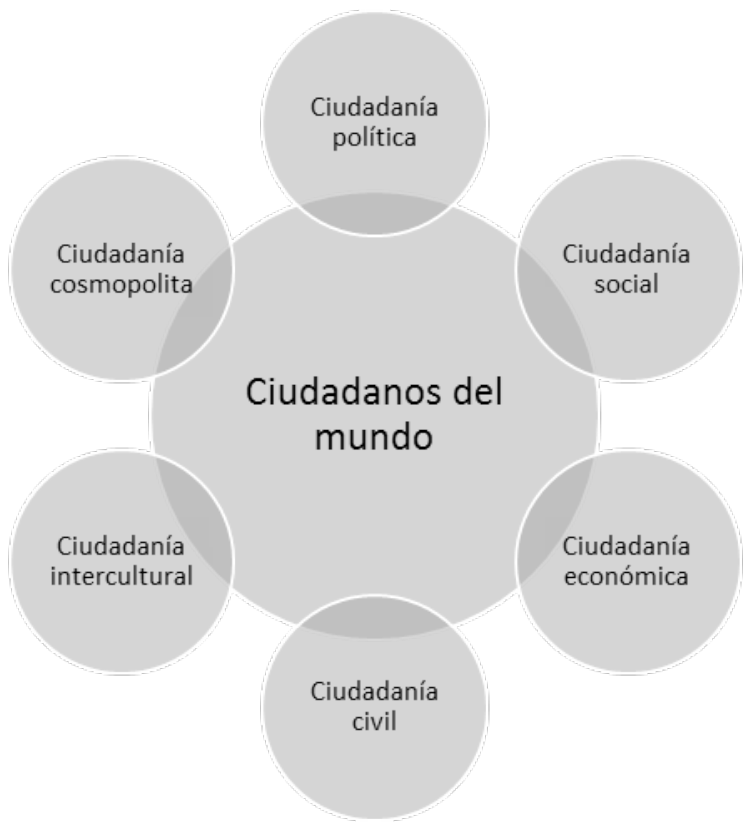

Fuente: Elaboración propia a partir de Cortina (2009). 
Estas dimensiones que integran la ciudadanía son evidencia de la versatilidad, transversalidad y plasticidad que debe tener la formación ciudadana en cualquier contexto de enseñanza, incluso en espacios de educación informal. Como ya se ha mencionado la ciudadanía legal o política identifica mediante estatutos políticos los derechos de los ciudadanos en el Estado del que forma parte, a la vez que la ciudadanía social reconoce a los miembros de una comunidad política y sus derechos básicos, independiente de si gocen o no de la categoría de ciudadanos legales.

La economía, independiente del modelo al que se haga referencia, tiene una dimensión pública y los ciudadanos tienen el derecho de recocerse como sujetos económicos que toman decisiones que impactan en su vida, en la de los otros y en el medioambiente. Esta interconexión también es visible en la ciudadanía civil, que alude a los lazos dados por la relación entre individuos con un fin común y que participan de los mismos espacios y áreas de interés, ya sea culturales, educativos o científicos.

En el contexto planetario de permanentes corrientes migratorias, es fundamental integrar una idea de ciudadanía intercultural, la que interpela directamente al derecho que tienen todos los miembros de una comunidad de experimentar, proteger y fomentar sus expresiones culturales, étnicas o propias de su identidad nacional o local. En una tensión positiva con la ciudadanía intercultural se encuentra la ciudadanía cosmopolita, caracterizada por trascender lo nacional y llevar la forma de vida democrática a un modo transnacional, caracterizada por la valoración de la diversidad.

El modelo de ciudadanía propuesto por Cortina (2009) que se ha descrito sintéticamente, es un importante referente en las creación del modelo teórico de esta investigación, vale destacar la relevancia que tiene la idea de una ciudadanía con identidad local, pero también habitante de una sociedad global, esto significa compartir un conjunto de elementos comunes como valores, normas de comportamiento, responsabilidades y acciones de participación en los asuntos que impactan a la sociedad en sus diferentes escalas, es por esta razón que, como plantea Pérez (2016): "la educación de la ciudadanía se complementa con una educación moral o ética, pues sin normas morales es imposible convivir en paz y respetar las libertades de todos" (p. 124).

Asimismo, la filosofía educativa de Dewey ya manifestaba la profunda relación que existe entre el sistema educativo y las instituciones en la sociedad contemporáneas. Podría decirse que las cualidades de un sistema educativo son muestra de cómo una sociedad busca formar sus nuevos miembros y como concibe su futuro. En ese contexto un modelo de educación ciudadana debe ser construido desde la participación democrática.

Hemos tenido ocasión de referirnos de pasada a la distinción entre la democracia como una idea de vida social y la democracia política como un sistema de gobierno. Evidentemente, ambas están relacionadas. La idea permanece estéril y vacía siempre que no se encarne en las relaciones humanas. Pero en la discusión hay que distinguirlas. La idea de democracia es una idea más amplia y más completa de los que se pueda ejemplificar en el Estado, aun en el mejor de los casos. Para que se realice, debe afectar a todos los modos de asociación humana, a la familia, a la escuela, a la industria, a la religión (Dewey, 2003, p. 135).

Dewey propuso potenciar la subjetividad de los individuos y facilitar el desarrollo de sus capacidades y potencialidades (Vergara y Martin, 2017). Esto suponía cuestionar 
la idea de que la democracia era solo un método compatible con cualquier forma de organización y estilo de vida. Una ciudadanía activa debe habitar en un sistema de democracia activa. Esta concepción de educación sostiene la necesidad de que la escuela desarrolle un individualismo democrático, una subjetividad política en la cual el individuo esté preparado para pensar por sí mismo, ser crítico, y como un miembro pleno, pueda ser parte de la sociedad.

Eis y Moulin-Doos (2017) distinguen una ciudadanía denominada liberal-democrática que abarca una doble dimensión en la que los ciudadanos son sujetos jurídicos titulares de derechos y al mismo tiempo son coactores políticos. "According to the liberal-individual dimension, a citizen, understood as a rights-holder, owns rights that he or she can defend individually before a court" ${ }^{2}$. Esta distinción es relevante porque ambas dimensiones no pueden estar disociadas en el proceso de enseñanza y aprendizaje de la formación ciudadana, es fundamental que el conocimiento sobre la ciudadanía se integre con el desarrollo de competencias y habilidades que le permitan a los ciudadanos ser coactores en las acciones políticas que los involucran, ya sea en escalas locales o globales.

En 2020, Contreras y Sánchez (2020) realizan un análisis de contenido a partir de ensayos sobre formación ciudadana, elaborados por estudiantes de pedagogía de la Universidad Católica Silva Henríquez. Uno de sus principales resultados en la dimensión de categorías emergentes, consistió en la construcción del carácter teleológico de esta disciplina, lo que fue denominado como la función pedagógica de la formación ciudadana y lo relevante de este hallazgo es que surge desde la mirada de los propios estudiantes en formación. Los resultados de ese estudio distinguieron que la función pedagógica de la formación ciudadana es también una función política, en la medida en que la ciudadanía debe ser educada para:

Resguardar la democracia, desarrollar el autorreconocimiento político y emocional, velar por el bien común, fomentar la deliberación como condición necesaria para las decisiones políticas y formar para analizar las ocurrencias de la vida cotidiana y comunitaria como expresiones de la vida política (Contreras y Sánchez, 2020, p. 345).

Los antecedentes teóricos aquí descritos, junto a los antecedentes empíricos especificados en la metodología, dieron paso a la construcción de un modelo teórico de formación ciudadana, que diera cuenta de diferentes enfoques de ciudadanía y por ende de sus contenidos y modos de enseñanza. El diseño del cuestionario para la descripción y conocimiento del pensamiento sobre formación ciudadana en estudiantes de pedagogía (Cuestionario PEU-FC) diversifica, amplía y complejiza la idea minimalista de la antigua educación cívica, al considerar el conocimiento cívico, el desarrollo de competencias y habilidades ciudadanas, y al integrar la educación en valores como parte de sus contenidos y prácticas, debido a que la interrelación de estas dimensiones convergen en la función pedagógico política de la educación ciudadana.

Según la dimensión democrática colectiva, los ciudadanos, entendidos como coactores, inician, codeciden y controlan las acciones políticas colectivas. Según la dimensión liberal-individual, el ciudadano, entendido como titular de derechos, posee derechos que puede defender individualmente ante un tribunal. 


\section{RESULTADOS Y DISCUSIÓN}

La primera fase de esta investigación correspondiente a la revisión de literatura, instrumentos de medición y política pública vigente sobre formación ciudadana en Chile generó los insumos para la operacionalización del instrumento (cuestionario) a partir de un objetivo, constructo, dimensiones y subdimensiones, que fueron traducidas en ítems.

La formación ciudadana, en su función político-pedagógica considera elementos que pueden ser reconocidos como de continuidad y cambio, respecto del modelo más tradicional que fue dominante hasta el siglo XX. El elemento de continuidad de dicho enfoque y que tiene más presencia en la actualidad es lo que se denominó como Conocimiento Cívico, es el contenido que ha sido más ampliamente abordado por la formación ciudadana, está relacionado con la apropiación teórica y conceptual del funcionamiento del sistema político del país incluido el sistema normativo y jurídico (Contreras y Sánchez, 2020; Cox y Castillo, 2015; Mardones, 2018). Cabe destacar que como elemento de innovación se incorporó en esta dimensión el conocimiento de los Derechos Humanos y también de los problemas propios de la sociedad actual, por estar íntimamente vinculados a la matriz política y jurídica de los Estados. Las siguientes dimensiones pueden ser identificadas como elementos de cambio respecto de la noción más clásica de la educación de la ciudadanía.

Una segunda dimensión de este modelo teórico que se consideró para la elaboración del Cuestionario PEU-FC son las llamadas Competencias y habilidades cívicas que se integran a la formación ciudadana como un modelo de ciudadano, un modelo de comportamiento y un modelo de sociedad, en el entendido de que la educación es la preparación para la vida social (Chaux et al., 2012; Contreras y Sánchez, 2020; Marina y Bernabeu, 2014) Son aquellas disposiciones que "permiten a los ciudadanos analizar y sintetizar información y argumentos, así como evaluar, llegar a conclusiones, tomar y defender posiciones sobre asuntos de interés público" (Haste, 2017, p. 216), ejemplo de ellas son: la participación ciudadana, la capacidad de resolución de conflictos, la autonomía personal, las destrezas de comunicación y la disposición para la cooperación y colaboración.

La tercera dimensión teórica de este modelo se identificó como Valores en Formación Ciudadana, puesto que "la educación de la ciudadanía, necesaria para el proceso de socialización del individuo y su participación en convivencia, es significada siempre en complemento con una dimensión ética o moral" (Contreras y Sánchez, 2020, p. 342). Los valores en formación ciudadana son disposiciones prácticas de la convivencia, entendiéndola como un aspecto esencial del bien común (Cortina, 2009); y como otros contenidos enseñados en el sistema escolar, pueden ser parte de procesos didácticos y evaluativos (Pérez, 2016).

Finalmente, en el Cuestionario PEU-FC se incorporó una cuarta dimensión para identificar elementos de convergencia o divergencia en el pensamiento que estudiantes de diferentes carreras del área de la educación poseen. Además, las carreras incorporadas son aquellas que están directamente vinculadas a la política pública vigente en Chile sobre formación ciudadana (Ley 20.911). Esta dimensión es Educación del profesorado en Formación Ciudadana y se refiere al conocimiento sobre formación ciudadana, asociado a las profesiones de Pedagogía en Historia y Geografía, Educación General Básica y Educación de Párvulos.

Para un conocimiento más detallado del resultado de esta fase de la investigación se puede observar la Tabla $\mathrm{N}^{\circ} 2$ que ilustra las dimensiones, subdimensiones y sus correspondientes definiciones conceptuales. 
Tabla 2. Síntesis del modelo teórico

\begin{tabular}{|c|c|c|}
\hline Dimensión: & Subdimensión: & Definición conceptual: \\
\hline \multirow{6}{*}{$\begin{array}{l}\text { Conocimiento } \\
\text { Cívico }\end{array}$} & Historia política del país & $\begin{array}{l}\text { Corresponde a la historia de Chile relacionada } \\
\text { con procesos, movimientos, líderes y acciones } \\
\text { políticas que han sido relevantes para el país. }\end{array}$ \\
\hline & Concepto de Ciudadanía & $\begin{array}{l}\text { Hace referencia a una amplia definición de } \\
\text { ciudadanía que integra componentes legales, } \\
\text { sociales, culturales y políticos. }\end{array}$ \\
\hline & $\begin{array}{l}\text { Constitución de la } \\
\text { República }\end{array}$ & $\begin{array}{l}\text { Alude al texto jurídico político que explicita el } \\
\text { rol del Estado y los derechos ciudadanos de la } \\
\text { República de Chile. }\end{array}$ \\
\hline & $\begin{array}{l}\text { Funcionamiento del sistema } \\
\text { político democrático }\end{array}$ & $\begin{array}{l}\text { Hace referencia a los procesos políticos que } \\
\text { garantizan el conocimiento de un sistema } \\
\text { democrático. }\end{array}$ \\
\hline & Derechos Humanos & $\begin{array}{l}\text { Conocimiento de los Derechos Humanos } \\
\text { contenidos en la Declaración Universal de los } \\
\text { Derechos Humanos. }\end{array}$ \\
\hline & $\begin{array}{l}\text { Problemas de la sociedad } \\
\text { actual, temas de interés } \\
\text { público }\end{array}$ & $\begin{array}{l}\text { Corresponde a las temáticas que son entendidas } \\
\text { como problemas de la sociedad contemporánea y } \\
\text { globalizada (cuidado del medioambiente, } \\
\text { migraciones, pobreza, etc.). }\end{array}$ \\
\hline \multirow{5}{*}{$\begin{array}{l}\text { Competencias } \\
\text { y habilidades } \\
\text { cívicas }\end{array}$} & Participación ciudadana & $\begin{array}{l}\text { Es el desarrollo del interés por involucrarse } \\
\text { activamente en la cultura ciudadana, a través de } \\
\text { instancias como el sufragio o mediante acciones } \\
\text { políticas que involucren la toma de decisiones } \\
\text { públicas. }\end{array}$ \\
\hline & Resolución de conflictos & $\begin{array}{l}\text { Habilidades para resolver problemáticas de } \\
\text { manera justa y pacífica, mediante mecanismos } \\
\text { como el diálogo. }\end{array}$ \\
\hline & Autonomía personal & $\begin{array}{l}\text { Capacidad de dirigir libremente la propia } \\
\text { conducta, de darse normas a uno mismo y que se } \\
\text { relacionen en equilibrio con la vinculación social. }\end{array}$ \\
\hline & Comunicación & $\begin{array}{l}\text { Se entiende como una capacidad fundamental } \\
\text { para la sociabilidad. Integra a las herramientas } \\
\text { lingüísticas el componente actitudinal de } \\
\text { expresión y comprensión en la transmisión de los } \\
\text { mensajes. }\end{array}$ \\
\hline & Cooperación y colaboración & $\begin{array}{l}\text { Capacidad de integrarse en el trabajo con otros y } \\
\text { para otros, situándose en el contexto y rol social. }\end{array}$ \\
\hline
\end{tabular}




\begin{tabular}{|c|c|c|}
\hline \multirow{3}{*}{$\begin{array}{l}\text { Valores en } \\
\text { Formación } \\
\text { Ciudadana }\end{array}$} & Educar en valores & $\begin{array}{l}\text { Considera un proceso de enseñanza y aprendizaje } \\
\text { donde los valores son cognoscibles y estimables, } \\
\text { por lo tanto enseñables y evaluables. }\end{array}$ \\
\hline & Valores indispensables & $\begin{array}{l}\text { Corresponde a los valores que una sociedad } \\
\text { establece como fundamentales para sostener el } \\
\text { sistema democrático y que articulan los } \\
\text { mecanismos de resolución de conflictos de } \\
\text { manera justa y pacífica. Se entiende que a partir } \\
\text { de ellos se articulan muchos más (libertad, } \\
\text { igualdad, solidaridad y respeto). }\end{array}$ \\
\hline & Ética y Moral & $\begin{array}{l}\text { Es el conocimiento que considera que toda } \\
\text { persona humana es inevitablemente moral y que } \\
\text { existe una ética cívica que es enseñable y } \\
\text { aprensible. }\end{array}$ \\
\hline $\begin{array}{l}\text { Educación del } \\
\text { profesorado en } \\
\text { Formación } \\
\text { Ciudadana }\end{array}$ & $\begin{array}{l}\text { Formación Ciudadana } \\
\text { asociada a las profesiones y } \\
\text { especialidades disciplinares }\end{array}$ & $\begin{array}{l}\text { Alude a la relación entre las disciplinas } \\
\text { específicas de formación de las carreras } \\
\text { estudiadas y la educación de la ciudadanía. }\end{array}$ \\
\hline
\end{tabular}

Fuente: Elaboración propia.

Ruiz (2014) señala que la operacionalización del constructo teórico es un proceso fundamental en la construcción de instrumentos de recogida de información como un cuestionario, puesto que implica traducir las dimensiones en elementos medibles; es decir, pasar de las dimensiones a los indicadores y de los indicadores a las preguntas o ítems.

El diseño final del cuestionario se caracteriza por un consentimiento informado que a su vez describe los aspectos centrales de la investigación, contextualiza su relevancia para la formación pedagógica, garantizando confidencialidad y anonimato en el uso de los datos. Las preguntas del cuestionario se contestan mediante escala de Likert. También se incluyó una pregunta abierta con el propósito de hacer un posterior trabajo de análisis de contenido.

Tabla 3. Ejemplos de muestra de los ítems que componen el Cuestionario PEU - FC

\begin{tabular}{|l|l|l|}
\hline Dimensión: & \multicolumn{1}{|c|}{ Subdimensión: } & \multicolumn{1}{c|}{ Ítem de ejemplo } \\
\hline \multirow{3}{*}{$\begin{array}{l}\text { Conocimiento } \\
\text { Cívico }\end{array}$} & Historia política del país & $\begin{array}{l}\text { Comprender que los gobiernos y sus actores } \\
\text { políticos forman parte de procesos históricos } \\
\text { amplios y complejos, es relevante para el } \\
\text { conocimiento cívico. }\end{array}$ \\
\cline { 2 - 3 } & $\begin{array}{l}\text { Constitución de la } \\
\text { República }\end{array}$ & $\begin{array}{l}\text { Es importante que la formación ciudadana enseñe } \\
\text { sobre la estructura que tiene la constitución, las } \\
\text { partes que la componen y los asuntos sobre los } \\
\text { cuales se manifiesta. }\end{array}$ \\
\hline
\end{tabular}




\begin{tabular}{|c|c|c|}
\hline \multirow{2}{*}{$\begin{array}{l}\text { Competencias } \\
\text { y habilidades } \\
\text { cívicas }\end{array}$} & Participación ciudadana & $\begin{array}{l}\text { La formación de la ciudadanía debe fomentar e } \\
\text { incentivar la participación en los sistemas de } \\
\text { organización civil (ONGs, fundaciones, } \\
\text { agrupaciones políticas, sindicatos, juntas de } \\
\text { vecinos, etc.) }\end{array}$ \\
\hline & Comunicación & $\begin{array}{l}\text { La capacidad de diálogo, escuchar al otro/a, en la } \\
\text { comunicación es una habilidad social } \\
\text { fundamental en la enseñanza de la Formación } \\
\text { Ciudadana para que niñas, niños y jóvenes } \\
\text { puedan resolver conflictos y crear consensos. }\end{array}$ \\
\hline \multirow{2}{*}{$\begin{array}{l}\text { Valores en } \\
\text { Formación } \\
\text { Ciudadana }\end{array}$} & Educar en valores & $\begin{array}{l}\text { Los valores son un tipo de contenido que se } \\
\text { enseña conjuntamente entre los núcleos } \\
\text { familiares y la Escuela por medio de la } \\
\text { Formación Ciudadana. }\end{array}$ \\
\hline & Valores indispensables & $\begin{array}{l}\text { La Formación Ciudadana debe proponer un } \\
\text { modelo de sociedad que valora y respeta la } \\
\text { diversidad social e interculturalidad. }\end{array}$ \\
\hline \multirow{2}{*}{$\begin{array}{l}\text { Educación del } \\
\text { profesorado en } \\
\text { Formación } \\
\text { Ciudadana }\end{array}$} & \multirow{2}{*}{$\begin{array}{l}\text { Formación Ciudadana } \\
\text { asociada a las profesiones y } \\
\text { especialidades disciplinares }\end{array}$} & $\begin{array}{l}\text { Niños y niñas de educación parvularia sí pueden } \\
\text { ser recibir enseñanza sobre la ciudadanía y la } \\
\text { convivencia en comunidad. }\end{array}$ \\
\hline & & $\begin{array}{l}\text { La Formación Ciudadana es un ámbito } \\
\text { disciplinar que puede ser abordado de forma } \\
\text { transversal en la enseñanza general básica. }\end{array}$ \\
\hline
\end{tabular}

Fuente: Elaboración propia.

Los ítems se crearon considerando criterios de diseño sugeridos por referencias de metodología de la investigación (Corral, 2010; Alcaraz et al., 2006; Casas Anguita et al., 2003): son breves para evitar confusiones en el encuestado, por lo mismo se buscó que fueran afirmaciones claras, precisas, con un lenguaje familiar al contexto de estudiantes de pedagogía, se evitó la formulación de preguntas en afirmación negativa o que significaran enjuiciamientos por parte del encuestado. Los ítems se presentan en orden a partir de secciones que coinciden con las dimensiones del modelo teórico.

En relación a la fase de validación, vale decir que corresponde al grado en que el proceso de pasar de lo teórico a lo empírico mide el concepto en cuestión (Ruiz, 2014). El Cuestionario PEU-FC se presentó a los jueces expertos con un total de 78 ítems y luego de su evaluación estos disminuyeron a 60. Los ítems eliminados causaron algunas adecuaciones menores en las subdimensiones del constructo, sin embargo esto no significó cambios en el modelo teórico que se propuso. 


\section{CONCLUSIONES}

De acuerdo con el objetivo de este artículo, es decir, dar a conocer resultados parciales de una investigación de carácter cuantitativo, principalmente las dimensiones conceptuales del modelo teórico utilizado para la construcción de un cuestionario y el resultado de la evaluación de contenido realizada a través de la validación de jueces expertos, es posible concluir que:

Las fases de trabajo de revisión de la literatura y posterior diseño y validación del Cuestionario PEU - FC han permitido identificar con amplia claridad la estructura de un modelo teórico sobre formación de la ciudadanía, que tiene la virtud de tener un enfoque transversal y multidimensional, junto con ser crisol de muchas ideas sobre educación de la ciudadanía que suelen ser más bien dispersas.

La dimensión de conocimiento cívico representa un elemento de continuidad del antiguo modelo de educación cívica que estuvo vigente en Chile hasta el período de la Dictadura cívico militar, en donde foco estaba puesto en la valoración de la institucionalidad y sistema de funcionamiento del Estado, la Constitución y el cumplimiento de normas jurídicas y sociales. Esta dimensión permanece en los contenidos de la Ley 20.911 y en la reforma curricular, sin embargo, se complejiza al integrar el estudio de los Derechos Humanos y los problemas de interés de la sociedad actual. Esto es significativo puesto que da equilibrio al conocimiento cívico al posicionarlo frente a los Derechos Humanos y al cuestionarlo permanentemente de frente a las problemáticas de interés de la sociedad actual, como por ejemplo los procesos migratorios, la protección del medioambiente, el desarrollo sustentable o la perspectiva de género, entre otros.

Actualmente la investigación sobre las temáticas abordadas en la educación de la ciudadanía está poniendo énfasis en enfoques anticoloniales (Sabzalian, 2019) y la lucha contra la injusticia racial (Clay y Rubin, 2020). El Cuestionario PEU-FC integra un enfoque intercultural, sin embargo, no se instala aún en el debate más complejo del pensamiento poscolonialista, puesto que en nuestra sociedad los debates están recientemente instalándose en la valoración de la diversidad étnica y la riqueza cultural que implican los procesos migratorios.

Como en todo proceso de enseñanza y aprendizaje, el conocimiento conceptual de lo cívico es solo una dimensión de los aprendizajes que requieren de aplicación en la convivencia para verse implementado, por este motivo es necesario que cualquier enfoque de formación de la ciudadanía integre el desarrollo de competencia y habilidades cívicas y sociales, por ejemplo la valoración y conocimiento del sistema democrático debe ser comprendido en la acción de la participación ciudadana, y para ello se necesita la práctica social. Lo mismo se puede evidenciar en asuntos como la resolución de conflictos o la cooperación y colaboración, son estas las instancias en que se despliegan las posibilidades de ejercer acciones cívicas que suelen estar nominadas como aspiraciones de una sociedad que busque la justicia social. Estudios contemporáneos, trabajan la acción deliberativa como una competencia fundamental para desarrollar en la educación ciudadana (Gibson, 2020; Ho y Barton, 2020) ya que esta allana el camino para que los jóvenes se integren a la sociedad civil y puedan influir en la política pública y en las acciones de los gobiernos.

La educación de la ciudadanía, entre sus diversos propósitos, fomenta la integración de niñas, niños y jóvenes a la sociedad y les permite el desarrollo de un pensamiento 
crítico en sus espacios de convivencia, a escala local y global, y conocer su comunidad a través de la educación en valores, que le da el dinamismo, la sitúa y hace promover reflexiones éticas y morales. Esto contribuye a la que la formación ciudadana sea un ámbito de la educación que huye del conocimiento monolítico y lo presiona a ajustarse, incluso fenomenológicamente, a la sociedad.

Asimismo, en este cuestionario se incluye la dimensión de Educación del profesorado en Formación Ciudadana puesto que es el escenario en que se enmarca la investigación, pero también con el propósito de fomentar la reflexión en sus propios protagonistas, de la relación que existe entre la formación profesional y el rol social que cumplirá ese trabajo en la práctica docente, puesto que son estos futuros profesores y profesoras lo que se verán interpelados a la educación de la ciudadanía.

Para el avance de esta investigación y posterior análisis de resultados finales será esencial el proceso de análisis de fiabilidad del instrumento, puesto que, considerando el enfoque del análisis de consistencia interna de los ítems, a través del cálculo de los coeficientes de alpha de Cronbach, es posible que los ítems se reduzcan, lo que aumente la aplicabilidad y replicabilidad del instrumento.

Este instrumento aspira a ser una herramienta innovadora de recogida de datos que insume información sobre el pensamiento de la educación de la ciudadanía en distintos actores de la comunidad educativa. En este caso es funcional para aportar a la formación del profesorado, pero también será útil, por ejemplo, para egresados de carreras de educación, estudiantes en práctica, profesores que estén insertos en el sistema e incluso encargados de gestión escolar. De todas formas es importante identificar las limitaciones de un instrumento propiamente cuantitativo, y es la necesidad de mixturar su análisis con el conocimiento del escenario en que se aplica, puesto que, en el caso de profesores en formación, son relevantes las características de sus planes de estudio, perfiles de egreso, sellos institucionales, entre otros, lo que puede hacer imprescindible el desarrollo de estrategias de investigación cualitativa para tensionar los eventuales resultados del Cuestionario PEU -FC.

El conocimiento sobre lo que se piensa por educación de la ciudadanía y su función político-pedagógica está en permanente transformación, responde a trayectos históricos, políticas de Estado y fenómenos locales y globales, por ello si la búsqueda de la justicia en nuestra sociedad descansa en una gran medida en la educación, la formación ciudadana de niñas, niños y jóvenes es clave, tanto para dar respuesta a sus inquietudes políticas como para aquellas comunidades en las que se insertan.

\section{REFERENCIAS BIBLIOGRÁFICAS}

Agencia de Calidad de la Educación. (2015). Estudio Internacional sobre Educación Cívica y Formación Ciudadana (ICCS): Marco de evaluación y ejemplos de preguntas. Agencia de Calidad de la Educación. http://archivos.agenciaeducacion.cl/Estudio_Marco_Evaluacion_ ICCS.pdf

(2019). Indicadores de desarrollo personal y social. https://www.agenciaeducacion.cl/ evaluaciones/indicadores-desarrollo-personal-social/

Alcaraz, F. G., Espín, A. A., Martínez, A. H. \& Alarcón, M. M. (2006). Diseño de Cuestionarios para la recogida de información: Metodología y limitaciones. Revista Clínica de Medicina de Familia, 1(15), 6.

Bordieu, P. y Passeron, J. C. (1996). La reproducción. Fontamara, México D. F. 
Estudios Pedagógicos XLVI N 3: 73-91, 2020

PENSAMIENTO SOBRE LA FORMACIÓN CIUDADANA EN ESTUDIANTES UNIVERSITARIOS, DIMENSIONES PARA LA CONSTRUCCIÓN DE UN CUESTIONARIO

Casas Anguita, J., Repullo Labrador, J. R. \& Donado Campos, J. (2003). La encuesta como técnica de investigación. Elaboración de cuestionarios y tratamiento estadístico de los datos (I). Atención Primaria, 31(8), 527-538.

Castillo Riquelme, V., Rodríguez Garcés, C. \& Escalona Burgos, J. (2018). Participación, vida democrática y sentido de pertenencia según tipo de establecimiento educativo en Chile. Páginas de Educación, 11(2), 108. https://doi.org/10.22235/pe.v11i2.1630

Chaux, E., Lleras, J. \& Velásquez, A. (2012). Competencias ciudadanas: De los estándares al aula: Una propuesta de integración a las áreas académicas. Ediciones Uniandes-Universidad de los Andes.

Clay, K. L. \& Rubin, B. C. (2020). "I look deep into this stuff because it's a part of me": Toward a critically relevant civics education. Theory and Research in Social Education, 48(2), 161-181. Scopus. https:// doi.org/10.1080/00933104.2019.1680466

Contreras, N. A. \& Sánchez, R. (2020). Formación ciudadana: significados emergentes en contexto de crisis social. Transformación, 16(3), 435-452.

Corral, Y. (2010). Diseño de cuestionarios para recolección de datos. Revista Ciencias de la Educación, 20(36). http://servicio.bc.uc.edu.ve/educacion/revista/n36/art08.pdf

Cortés, Á. C., Gómez, J. F. C. \& López, J. M. V. (2016). La educación para la ciudadanía en la Unión Europea: perspectivas supranacional y comparada. Journal of Supranational Policies of Education (JoSPoE), 5, Article 5. https://revistas.uam.es/jospoe/article/view/6664

Cortina, A. (2009). Ciudadanos del mundo: Hacia una teoría de la ciudadanía - Tercera edición. Alianza Editorial.

Cox, C., Bascopé, M., Castillo, J., Miranda, D. \& Bonhomme, M. (2014). Educación Ciudadana en América Latina: Prioridades de los Currículos Escolares. IBE UNESCO Working Papers on Curriculum Issues, 1-41.

Cox, C. \& Castillo, J. C. (2015). Aprendizaje de la ciudadanía: Contextos, experiencias y resultados. Ediciones UC.

Dewey, J. (2003). Viejo y nuevo individualismo. Paidós.

Eis, A. \& Moulin-Doos, C. (2017). Cosmopolitan citizenship education: Realistic political program or program to disillusioned powerlessness? A plea for a critical power perspective within global citizenship education. Journal of Social Science Education, 16(4), 49-59. Scopus. https://doi.org/10.4119/UNIBI/ jsse-v16-i4-1639

Fierro, J. (2016). La ciudadanía y sus límites. Editorial Universitaria.

Gibson, M. (2020). From deliberation to counter-narration: Toward a critical pedagogy for democratic citizenship. Theory and Research in Social Education, 48(3), 431-454. Scopus. https://doi.org/10.108 0/00933104.2020.1747034

Haste, H. (2017). Nueva ciudadanía y educación. Grupo Planeta.

Ho, L.-C. \& Barton, K. C. (2020). Preparation for civil society: A necessary element of curriculum for social justice. Theory and Research in Social Education. Scopus. https://doi.org/10.1080/00933104 .2020 .1763880

Lawshe, C. H. (1975). A quantitative approach to content validity. Personnel Psychology, 28(4), 563-575. https://doi.org/10.1111/j.1744-6570.1975.tb01393.x

Magendzo,A. \& Pavez, J. (2016). Derechos humanos en los lineamientos curriculares referidos a la formación ciudadana. Praxis Educativa, 20(1), 13-27. https://doi.org/10.19137/praxiseducativa-2016-200102

Mardones, R. (2018). Las controversias políticas de la educación ciudadana. En I. Sánchez (Ed.), Ideas en Educación II. Definiciones en Tiempos de Cambio. Ediciones Universidad Católica de Chile. https:// www.researchgate.net/publication/326468729_Las_controversias_politicas_de_la_educacion_ ciudadana

Marina, J. A. \& Bernabeu, R. (2014). Competencia social y ciudadana. Alianza Editorial.

Núñez, K. (2017). Evaluación de los aprendizajes sobre ciudadanía: Meta evaluación de los instrumentos utilizados en el segundo ciclo básico chileno. Estudios pedagógicos (Valdivia), 43(2), 253-276. https://doi.org/10.4067/S0718-07052017000200014 
Nurdin, E. S. (2017). Civic Education policies: Their effect on university students' spirit of nationalism and patriotism. Citizenship, Social and Economics Education, 16(1), 69-82. Scopus. https://doi. org/10.1177/2047173416688039

Orellana Fonseca, C. \& Muñoz Labraña, C. (2019). Escuela y Formación ciudadana: Concepciones de ciudadanía, formación ciudadana y del rol de la escuela. Revista Electrónica Interuniversitaria de Formación del Profesorado, 22(2),137-149.

Parji \& Feriandi, Y. A. (2020). Development of Student Participation Instruments in Politics for Civic Education Learning in the University. Universal Journal of Educational Research, 8(8), 36793689. Scopus. https://doi.org/10.13189/ujer.2020.080847

Pérez, C. P. (2016). Educación en valores para la ciudadanía: Estrategias y técnicas de aprendizaje. Desclée de Brouwer.

PNUD. (2018). Estudio sobre la puesta en marcha del Plan de Formación Ciudadana. PNUD. http:// www.ciudadaniayescuela.cl/wp-content/uploads/2018/04/Estudio-puesta-en-marcha-del-Plande-Formaci\%C3\%B3n-Ciudadana.pdf

Rancière, J. (2014). El maestro ignorante. Cinco lecciones sobre la emancipación intelectual. Hueders Educación.

Ruiz, A. (2014). La operacionalización: De elementos teóricos al proceso de medida. http://diposit. ub.edu/dspace/handle/2445/53152

Sabzalian, L. (2019). The tensions between Indigenous sovereignty and multicultural citizenship education: Toward an anticolonial approach to civic education. Theory and Research in Social Education, 47(3), 311-346. Scopus. https://doi.org/10.1080/00933104.2019.1639572

Savater, F. (2007). Política para Amador. Grupo Planeta (GBS).

Schulz, W., Ainley, J., Fraillon, J., Kerr, D. \& Losito, B. (2010a). ICCS 2009 International Report: Civic knowledge, attitudes, and engagement among lower- secondary school students in 38 countries (p. 314). International Association for the Evaluation of Educational Achievement.

Schulz, W., Ainley, J., Fraillon, J., Kerr, D. \& Losito, B. (2010b). Resultados iniciales del Estudio Internacional de Educación Cívica y Ciudadana de la IEA (p. 117). Agencia Internacional para la Evaluación del Rendimiento Educativo.

Schulz, W., Fraillon, J., Ainley, J., Losito, B. \& Kerr, D. (2010). Estudio internacional sobre educación cívica y ciudadana. Marco de la evaluación. Agencia Internacional para la Evaluación del Rendimiento Educativo. https://www.iea.nl/sites/default/files/2019-04/ICCS_2009_Framework_ Spanish.pdf

Serrano, S. (2018). El liceo: Relato, memoria, política. Penguin Random House Grupo Editorial.

Touraine, A. (2015). ¿Qué Es la Democracia? Fondo de Cultura Económica.

Unidad Currículum y Evaluación del Ministerio de Educación de Chile. (2020a). $3^{\circ}$ y $4^{\circ}$ Medio FG /Educación ciudadana 3o medio. https://www.curriculumnacional.cl/. https://www. curriculumnacional.cl/docentes/Formacion-General/Educacion-ciudadana/Educacion-ciudadana3-medio/140122:Programa-FG-Educacion-ciudadana-3-medio

Unidad Currículum y Evaluación del Ministerio de Educación de Chile. (2020b). $3^{\circ}$ y $4^{\circ}$ Medio FG /Educación ciudadana 4o medio. https://www.curriculumnacional.cl/. https://www. curriculumnacional.cl/docentes/Formacion-General/Educacion-ciudadana/Educacion-ciudadana4-medio/140124:Programa-FG-Educacion-ciudadana-4-medio

Vergara, J. y Martin, A. (2017). Pensar la educación, desde Friedman a Dewey. Editorial Universitaria, Santiago, Chile. 
